Research Paper

\title{
Neuronal Effects of Sugammadex in combination with Rocuronium or Vecuronium
}

\author{
Martin Aldasoro ${ }^{1}$, Adrian Jorda ${ }^{1}$, Constanza Aldasoro², Patricia Marchio', Sol Guerra-Ojeda1, Marc

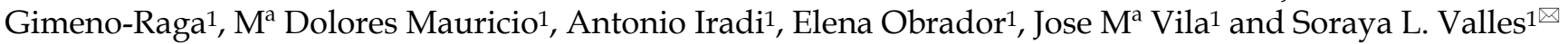 \\ 1. Department of Physiology, School of Medicine, University of Valencia, Valencia, Spain; \\ 2. Hospital General de Castellon, Castellon, Spain. \\ $\triangle$ Corresponding author: Soraya L. Valles. Department of Physiology, School of Medicine, University of Valencia. Blasco Ibañez 15, Valencia, Spain. Tel: +34 \\ 963983 813; Fax: +34 963864 646. Email: lilian.valles@uv.es. \\ (C) Ivyspring International Publisher. This is an open access article distributed under the terms of the Creative Commons Attribution (CC BY-NC) license \\ (https://creativecommons.org/licenses/by-nc/4.0/). See http://ivyspring.com/terms for full terms and conditions.
}

Received: 2016.09.12; Accepted: 2016.12.28; Published: 2017.02.23

\begin{abstract}
Rocuronium (ROC) and Vecuronium (VEC) are the most currently used steroidal non-depolarizing neuromuscular blocking (MNB) agents. Sugammadex (SUG) rapidly reverses steroidal NMB agents after anaesthesia. The present study was conducted in order to evaluate neuronal effects of SUG alone and in combination with both ROC and VEC. Using MTT, CASP-3 activity and Western-blot we determined the toxicity of SUG, ROC or VEC in neurons in primary culture. SUG induces apoptosis/necrosis in neurons in primary culture and increases cytochrome $\mathrm{C}(\mathrm{CytC}$ ), apoptosis-inducing factor (AIF), Smac/Diablo and Caspase 3 (CASP-3) protein expression. Our results also demonstrated that both ROC and VEC prevent these SUG effects. The protective role of both ROC and VEC could be explained by the fact that SUG encapsulates NMB drugs. In BBB impaired conditions it would be desirable to control SUG doses to prevent the excess of free SUG in plasma that may induce neuronal damage. A balance between SUG, ROC or VEC would be necessary to prevent the risk of cell damage.
\end{abstract}

Key words: rocuronium, vecuronium, sugammadex, neuron, apoptosis.

\section{Introduction}

NMB drugs are used to obtain skeletal muscle relaxation in order to enable endotracheal intubation, facilitate mechanical ventilation and prevent patient from moving to assure optimal surgical conditions $[1,2]$. ROC and VEC are the most currently used steroidal non-depolarizing MNB agents [1,3,4,5] acting as competitive antagonists to acetylcholine (Ach) for binding to the nicotinic receptor at the neuromuscular junction $[6,7,8]$. After tracheal extubation, residual NMB may result in pulmonary complications, airway obstruction and increased morbidity. In this sense, pharmacological reversion of NMB is a useful tool to prevent post-anaesthetic complications $[9,10]$. Sugammadex (SUG) is a modified $\gamma$-cyclodextrin that rapidly reverses steroidal neuromuscular blockade after anaesthesia without inhibition of the enzyme acetylcholinesterase
$[11,12,2]$. It is a drug with a doughnut-like molecular structure containing a lipophilic core and a hydrophilic periphery forming a complex with ROC or VEC that reduces their binding affinity to nicotinic receptors in the neuromuscular junctions [13]. SUG combined with ROC or VEC is well tolerated with no clinical evidence of residual neuromuscular blockade at recommended doses of SUG [14,5].

In a previous study we demonstrated that sugammadex induces apoptosis/necrosis in neurons in primary culture and increases cytochrome C (CytC), apoptosis-inducing factor (AIF), Smac/Diablo and Caspase 3 (CASP-3) protein expression [15]. However, the combination of SUG with ROC or VEC has not been previously assessed. The present study was conducted in order to evaluate neuronal effects of SUG in combination with ROC or VEC 


\section{Material and Methods}

\section{Materials}

All animals were handled according to the recommendations of the Bioethics Committee of the School of Medicine of the University of Valencia, Spain. Ethics committee specifically approved this study (number A1265026030697). SUG was acquired from Merck Millipore products (Madrid, Spain). Rocuronium Bromide and Vecuronium Bromide were obtained from Sigma Aldrich (Madrid, Spain). Appropriate concentrations of SUG $(75 \mu \mathrm{g} / \mathrm{ml})$, ROC $(2 \mu \mathrm{g} / \mathrm{ml})$ or VEC $(0.33 \mu \mathrm{g} / \mathrm{ml})$ were used for these experiments. Dulbecco's modified Eagle's medium (DMEM) and foetal bovine serum (FBS) were obtained from Gibco life technology (Gibco Invitrogen Corporation, Barcelona, Spain). 3-(4,5-dimethyl-2thiazolyl)-2,5-dipheniyl-2H-tetrazolium bromide (MTT) was purchased from Sigma Chemical Co. (St Louis, MO). Western blot enhanced chemoluminescent detection system (ECL) was obtained from Amersham Bioscience (Amersham Biosciences, Barcelona, Spain). Monoclonal anti-glial fibrillary acidic protein (anti-GFAP) antibody (1:500) was acquired from Sigma-Aldrich Biotech (Madrid, Spain). Monoclonal anti-MAP-2 protein (anti-MAP-2) antibody (1:500) and monoclonal anti-cytochrome C protein (anti-CytC) antibody (1:500) and anti- $\alpha$ tubulin were acquired from Santa Cruz Biotechnology (Barcelona, Spain). Anti-apoptosis-inducing factor (anti-AIF) (1:500) and anti-Smac/Diablo (1:500) were obtained from Sigma-Aldrich Biotech. (Barcelona, Spain). All other reagents were of analytical or culture grade purity.

\section{Neurons in primary culture}

Primary cultures of cortical neurons were prepared from the cerebral cortex of 14-15-day old rat foetuses ( 5 female rats to obtain the foetuses). The cerebral cortex, obtained under toxin-free sterile conditions, was dissected and dissociated mechanically by pipetting 10 times with DMEM (10 $\mathrm{ml}$ for the cortex obtained from 12-14 foetuses). The cell suspension was filtered through a nylon mesh with a pore size of $90 \mu \mathrm{m}$ and plated $\left(5 \times 10^{4}\right.$ cells $/ \mathrm{cm}^{2}$ ) on poly-lysine-coated dishes. After the cell attachment $(1 \mathrm{~h})$, the plating medium was changed to DMEM pH 7.4, supplemented with $10 \%$ foetal bovine serum (FBS), $10 \mathrm{mM}$ HEPES, $40 \mathrm{mM} \mathrm{NaHCO}$, 100 units $/ \mathrm{ml}$ penicillin, and $100 \mathrm{mg} / \mathrm{ml}$ streptomycin. One hour is enough to stick neurons and to avoid astrocytes sticking on the plate.

After 4 days, the plating medium was changed with another medium prepared as described above. At seven days of culture, 20\% FBS was changed to a
$10 \%$ solution. Cultures were grown in a humidified atmosphere of $5 \% \mathrm{CO}_{2}$ and $95 \% \mathrm{O}_{2}$, at $37^{\circ} \mathrm{C}$, for 3 days, and then exposed to cytosine $\beta$-D arabinofuranoside $(10 \mu \mathrm{M})$ for $24 \mathrm{~h}$ to prevent proliferation of no neuronal cells. Possible contamination by astrocytes was assessed by immuno-fluorescence using monoclonal anti-glial fibrillary acidic protein (GFAP) (astrocytes marker: Sigma-Aldrich. 1:500) antibody (clone G-A-5) and polyclonal anti-microtubuleassociated protein 2 (MAP-2) (neuronal marker: Sigma-Aldrich. 1:500). Under these conditions approximately $99 \% \pm 3 \%$ of all cultured cells were neurons [15]. All animal work was performed according to minimize animal suffering and to reduce the total number of animals used.

\section{MTT assay}

Cellular viability of the cultures was determined by MTT assay [3-(4,5-dimethylthiazol-2-yl)-2,5diphenyltetrazolium bromide] [16]. Cells were plated in a 96 well culture plate and incubated with SUG, ROC, VEC, SUG+ROC and SUG+VEC during $24 \mathrm{~h}$. After cell treatments, the medium was removed and the cortical cells were incubated with red free medium and MTT solution $[0.5 \mathrm{mg} / \mathrm{ml}$, prepared in a phosphate buffer saline (PBS) solution] for $4 \mathrm{~h}$ at $37^{\circ} \mathrm{C}$. Finally the medium was removed and formazan particles were dissolved in dimethyl sulfoxide (DMSO). Cell viability was normalized relative to control cells whose viability was noted as $100 \%$ when treated for the indicated periods with the corresponding vehicles. Relative amount of MTT reduction, was determined by spectrophotometry at $570 \mathrm{~nm}$.

\section{Lactate Dehydrogenase (LDH) Assay}

To evaluate plasma membrane integrity, LDH release was determined by monitoring the leakage of the cytosolic LDH to the extracellular medium. LDH was measured spectrophotometrically at $340 \mathrm{~nm}$, following the rate of conversion of reduced nicotinamide adenine dinucleotide to oxidized nicotinamide adenine dinucleotide.

\section{Optic microscopy}

Neuron monolayers growing on culture dishes were used for optic microscopy studies. Photographs were made at the seventh day of culture. SUG, ROC and VEC were added $24 \mathrm{~h}$ before those images were obtained.

\section{Caspase 3 activity assay}

Sub-cellular fractioning of cortical neurons was carried out to segregate cytosol and membrane sections. Briefly, cells in the supernatant were collected by centrifugation and attached cells scraped 
in $5 \mathrm{mM}$ EDTA in PBS were pelleted by a $10 \mathrm{~min}, 4^{\circ} \mathrm{C}$ centrifugation at $1000 \times \mathrm{g}$. Pellets were re-suspended in $200 \mu \mathrm{l}$ of fractionation buffer $(0.25 \mathrm{M}$ sucrose, $1 \mathrm{mM}$ Mg chloride, $2 \mathrm{mM}$ EGTA, and $25 \mathrm{mM}$ HEPES, $\mathrm{pH}$ 7.4) and lysed by three cycles of flash freezing in liquid nitrogen. Lysates were then centrifuged at $100000 \times \mathrm{g}$ for $30 \mathrm{~min}$ at $4^{\circ} \mathrm{C}$. Supernatants (cytosol fraction) were separated and the pellet (membrane fraction) was dissolved again in $200 \mu \mathrm{l}$ of fractionation buffer containing 5\% Triton X-100 and briefly sonicated. Whole-cell lysates were obtained by harvesting or homogenizing the samples in sub-cellular fractioning buffer containing $2.5 \%$ Triton X-100, followed by a brief sonication. This activity was measured in cytosolic fractions by using a highly sensitive colorimetric substrate, N-acetyl-Asp-GluVal-Asp p-nitroanilide (Ac-DEVD-pNA) following the manufacturer's instructions (CalBiochem, La Jolla, CA). Enzyme activity ( $\mathrm{pmol} / \mathrm{min}$ ) was calculated, using the manufacturer's formulae.

\section{Western-blots}

Attached cells (neurons in primary culture) were collected and harvested with $\mathrm{pH} 7.4$ ice-cold buffer (0.125 M Tris- $\mathrm{HCl}, \mathrm{pH} 6.8,2 \%$ SDS, 19\% glycerol, 1 $\mathrm{mM}$ phenylmethylsulfonyl fluoride, $1 \mu \mathrm{g} / \mathrm{ml}$ leupeptin, $1 \mu \mathrm{g} / \mathrm{ml}$ aprotinin, and $1 \mu \mathrm{g} / \mathrm{ml}$ pepstatin), and disrupted by repeated cycles of freezing and thawing, and then boiled for $5 \mathrm{~min}$ at $90^{\circ}$. Cell extracts were homogenized and centrifuged $(10000 \times \mathrm{g}$ for 10 $\min$ at $4^{\circ} \mathrm{C}$ ). The resulting supernatant was used for western-blot analysis. Protein concentration was determined using a modified Lowry method [17]. Proteins were separated by SDS-PAGE gels and transferred to nitrocellulose membranes using standard techniques. Membranes were blocked with $5 \%$ dried milk in TBS containing $0.05 \%$ Tween-20 and then incubated with the corresponding antibodies following the manufacturer's recommendations. The blots were washed three times, 15 minutes each cycle, with phosphate-buffered saline containing $0.2 \%$ Tween 20 and then incubated for $1 \mathrm{~h}$ with a secondary horseradish peroxidase-linked anti-rabbit or antimouse IgG antibody. ECL was used for antibody detection. Auto-radiographic signals were assessed using a Bio-Rad scanning densitometer.

\section{Statistical significance}

Statistical analyses were performed using the t-Student test and one way ANOVA with the program GraphPad Prism 7. $\mathrm{p} \leq 0.05$ was considered statistically significant.

\section{Results}

\section{SUG, ROC, VEC and cell viability}

The role of SUG, ROC and VEC on cell viability was studied using MTT conversion assays. Figure 1 shows that incubation with SUG $(75 \mu \mathrm{g} / \mathrm{ml})$ at different treatment times $(6,12$ and $24 \mathrm{~h})$ induced a significant decrease in neuron viability at any time tested compared with control cells. We choose for future experiments $24 \mathrm{~h}$ treatment time, because it represents the biggest decrease data compared to control neurons.

In Figure 2, we show that ROC or VEC did not change cell viability compared to control cells. On the other hand, both ROC and VEC prevented the decrease in cell viability induced by SUG (Figure 2).

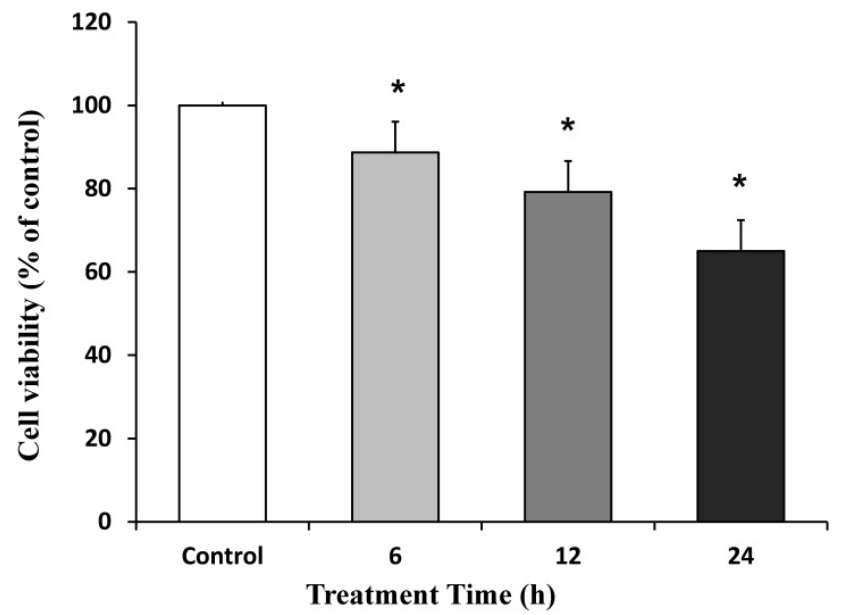

Figure 1. Neuronal cell death induced by SUG $(75 \mu \mathrm{g} / \mathrm{ml})$. Time dependent neurotoxicity of SUG was determined by the MTT assay. Data are means \pm SD for 4 independent experiments. *p $\leq 0.05$ comparing SUG vs control (no additions)

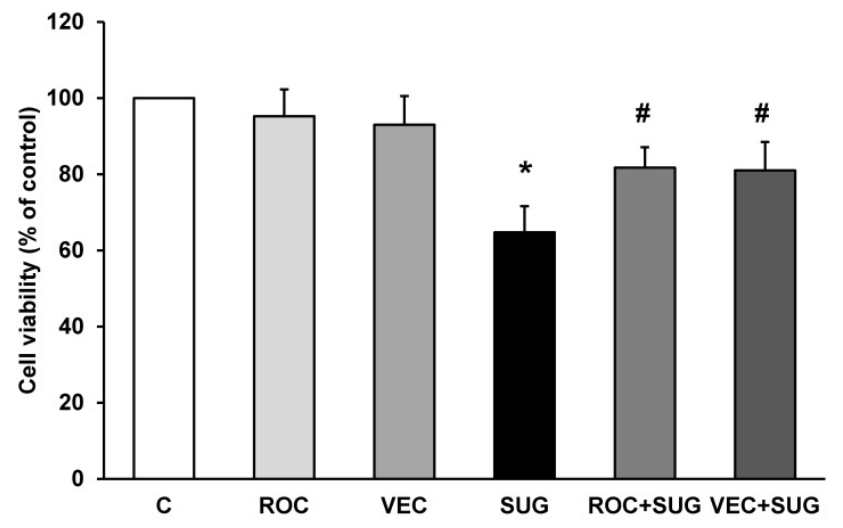

Figure 2. Determination of cell viability using ROC $(2 \mu \mathrm{g} / \mathrm{ml})$, VEC $(0.33$ $\mu \mathrm{g} / \mathrm{ml})$, SUG $(75 \mu \mathrm{g} / \mathrm{ml})$, ROC+SUG $(2 \mu \mathrm{g} / \mathrm{ml}$ and $75 \mu \mathrm{g} / \mathrm{ml}$ respectively), and VEC+SUG $(0.33 \mu \mathrm{g} / \mathrm{ml}$ and $75 \mu \mathrm{g} / \mathrm{ml}$ respectively $)$ by the MTT assay. ${ }^{*} \mathrm{p} \leq 0.05 \mathrm{vs}$ control. ${ }^{p} \leq 0.05$ vs SUG. Data are means \pm SD for 5 independent experiments. 
ROC and VEC did not increase LDH release whereas neurons incubated with SUG for $24 \mathrm{~h}$ showed a significant LDH leakage of $61.2 \%$ compared to control cells. The combination of SUG with ROC or $\mathrm{VEC}$ did not increase $\mathrm{LDH}$ release, indicating that ROC and VEC prevent cell death induced by SUG (Figure 3).

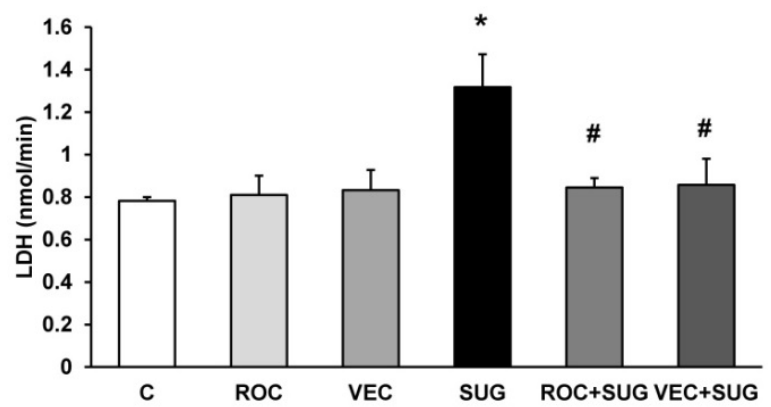

Figure 3. LDH assay using ROC $(2 \mu \mathrm{g} / \mathrm{ml})$, VEC $(0.33 \mu \mathrm{g} / \mathrm{ml})$, SUG $(75 \mu \mathrm{g} / \mathrm{ml})$, ROC+SUG $(2 \mu \mathrm{g} / \mathrm{ml}$ and $75 \mu \mathrm{g} / \mathrm{ml}$ respectively), and VEC+SUG $(0.33 \mu \mathrm{g} / \mathrm{ml}$ and $75 \mu \mathrm{g} / \mathrm{ml}$ respectively). ${ }^{*} \mathrm{p} \leq 0.05$ vs control. \#p $\leq 0.05$ vs SUG. Data are means \pm SD for 4 independent experiments.

\section{Optic microscopy after SUG, ROC and VEC treatment}

Using optic microscopy technique, we detected a decrease in cell number after $24 \mathrm{~h}$ of SUG treatment (Figure 4B) compared to control neurons (Figure 4A). The presence of ROC or VEC prevented the decrease in the number of neurons induced by SUG, Figure $4 \mathrm{C}$ and D.

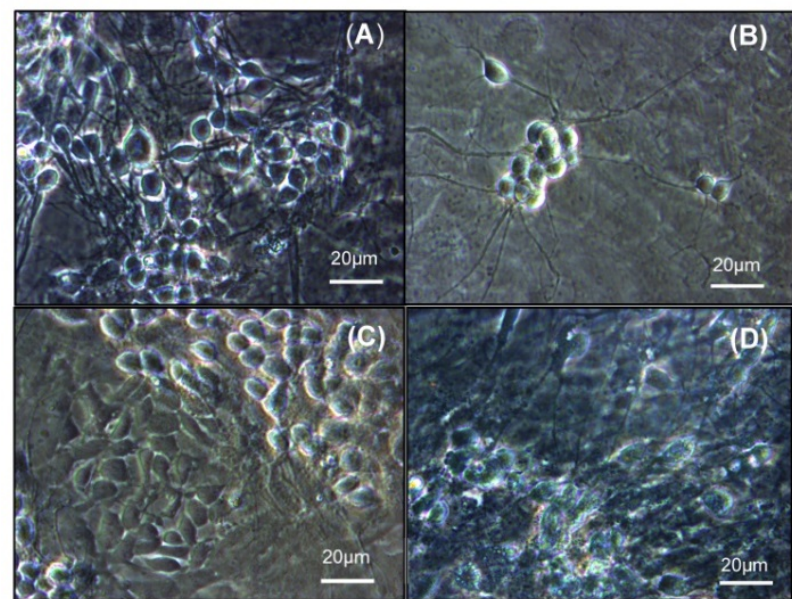

Figure 4. Optic microscopic image of cultured neurons incubated without drugs (A), with SUG $(75 \mu \mathrm{g} / \mathrm{ml})(B)$, ROC $(2 \mu \mathrm{g} / \mathrm{ml})+$ SUG $(75 \mu \mathrm{g} / \mathrm{ml})(\mathrm{C})$ and $\operatorname{VEC}(0.33 \mu \mathrm{g} / \mathrm{ml})+$ SUG $(75 \mu \mathrm{g} / \mathrm{ml})(D)$.

\section{Caspase 3 activity}

Neither ROC nor VEC altered the caspase 3 activity. The presence of SUG $(75 \mu \mathrm{g} / \mathrm{ml})$ for $24 \mathrm{~h}$ increased caspase 3 activity from 3.5 (control cells) to $6.89 \mathrm{pmol} / 10^{6}$ cells $\mathrm{x} \mathrm{min}$, indicating significant increase of apoptosis after SUG addition. In the culture medium ROC or VEC avoided caspase-3 activity increase produced by SUG (Figure 5), which indicated a prevention of apoptosis when either ROC or VEC were present in the culture.

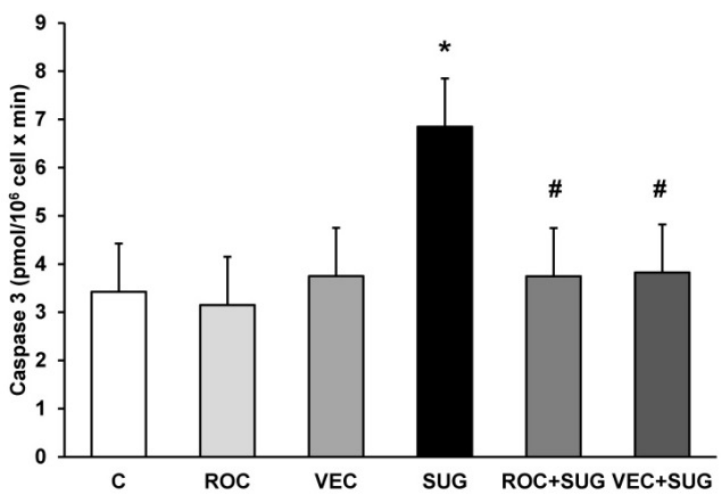

Figure 5. Determination of caspase 3 activity without $(\mathrm{C})$ and with ROC $(2 \mu \mathrm{g} / \mathrm{ml})$, VEC $(0.33 \mu \mathrm{g} / \mathrm{ml})$, SUG $(75 \mu \mathrm{g} / \mathrm{ml})$, ROC+SUG $(2 \mu \mathrm{g} / \mathrm{ml}$ and $75 \mu \mathrm{g} / \mathrm{ml}$ respectively), and VEC+SUG $\left(0.33 \mu \mathrm{g} / \mathrm{ml}\right.$ and $75 \mu \mathrm{g} / \mathrm{ml}$ respectively). ${ }^{*} \mathrm{p} \leq 0.05$ vs control. \# $\mathrm{P} \leq 0.05$ vs SUG. Data are means \pm SD for 4 independent experiments.

\section{Proteins related to cell-death molecular signals}

Using western-blot technique shown in Figures 6, 7 and 8 different protein levels related to cell-death molecular signals were determined. ROC and VEC did not affect cytochrome c expression in neurons in primary culture. Nevertheless, SUG produced an increase of cytochrome $c$ expression compared with control neurons (Figure 6). SUG incubated with either ROC or VEC avoided cytochrome c overexpression induced by SUG.
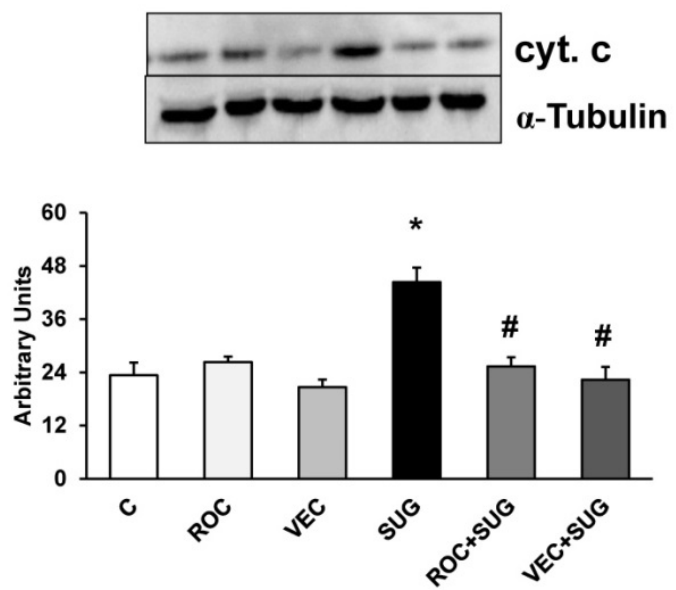

Figure 6. Quantitative analysis of cytochrome c. Results are expressed as relative changes in arbitrary units normalized against an internal standard ( $\alpha$-tubulin). Data are means \pm SD (error bars) $(n=5)$. *p $\leq 0.05$ vs control; \#p $\leq$ 0.05 vs SUG. 
Figure 7 shows Smac/Diablo expression in neurons in primary culture. ROC and VEC did not modify Smac/Diablo expression whereas SUG increased this protein. The presence of either ROC or VEC prevented Smac/Diablo overexpression induced by SUG.
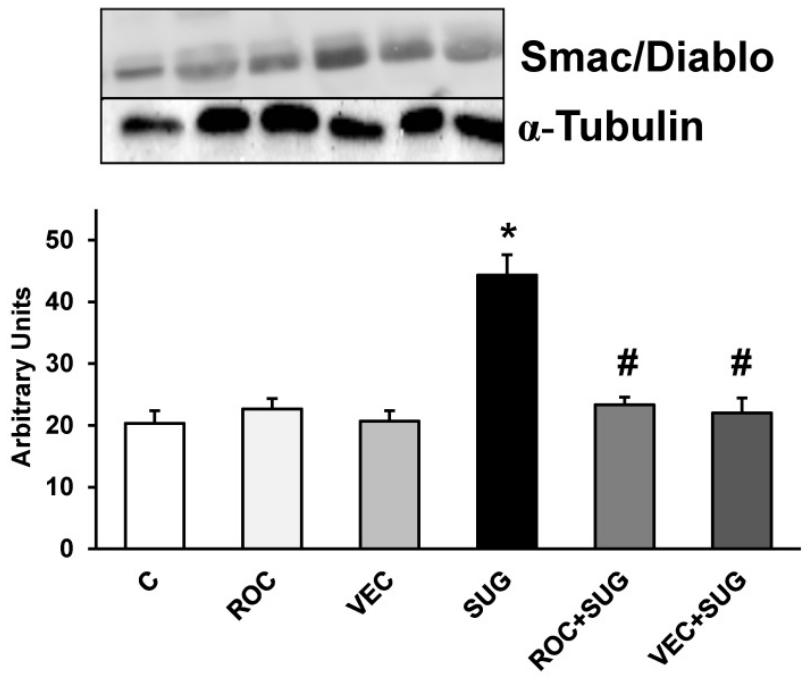

Figure 7. Results (Smac/Diablo) of quantitative analysis of immunoblots are means $\pm S D$ (error bars) $(n=4)$ expressed as relative changes in arbitrary units normalized against an internal standard ( $\alpha$-tubulin). ${ }^{*} p \leq 0.05$ vs control; ${ }^{*} p \leq$ 0.05 vs SUG.

We demonstrated in Figure 8 the increase in AIF protein expression after SUG addition compared to control cells that was prevented by co-incubation with either ROC or VEC. Neither ROC nor VEC alone managed to modify AIF expression.
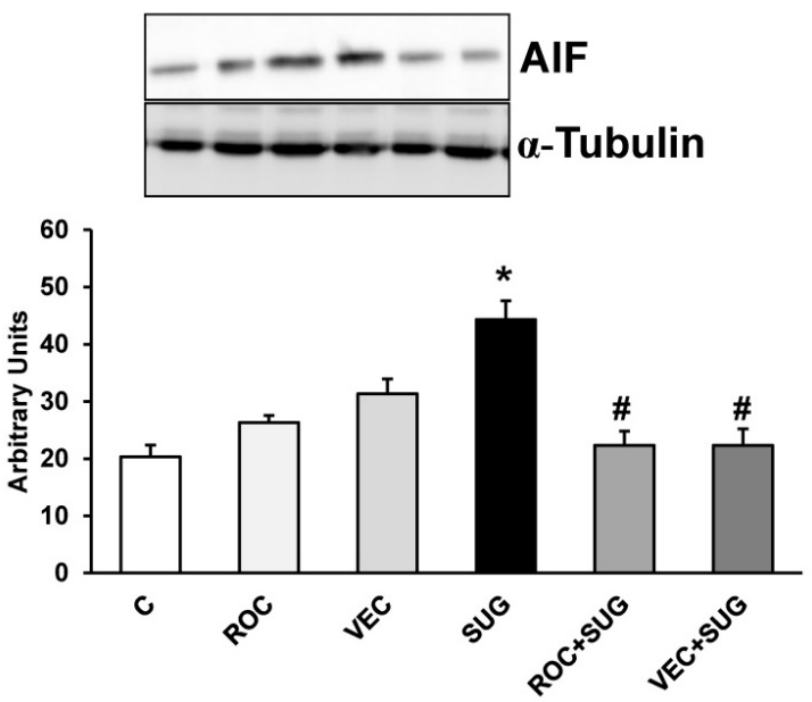

Figure 8. Results (AIF) of quantitative analysis of immunoblots are means $\pm S D$ (error bars) $(n=5)$ expressed as relative changes in arbitrary units normalized against an internal standard ( $\alpha$-tubulin). ${ }^{*} \mathrm{p} \leq 0.05$ vs control; ${ }^{*} \mathrm{p} \leq 0.05$ vs SUG.

\section{Discussion}

We previously demonstrated that SUG at clinically significant doses, induces neuronal death by apoptosis/necrosis, increasing CytC, AIF, Smac/Diablo expression and CASP-3 activity [15]. In the present work, we show how ROC and VEC did not induce any direct effect in neurons in primary culture, but prevented toxic neuronal effects induced by SUG.

ROC and VEC act as antagonists of the cholinergic receptor at neuromuscular junction and this effect is reversed by SUG [11]. The side chains of SUG bind to steroidal NMB $[18,19]$. The union is non-covalent with Van-der-Waals interactions playing a minor role, and positively charged groups of NMB electrostatically bind negatively charged end groups of SUG [18]. The encapsulation by SUG, forming a 1:1 complex with free intravascular steroidal NMB drugs, rapidly reduces the concentration of both ROC and VEC in the neuromuscular junction, resulting in an earlier reversal of the neuromuscular blockade [5]. In a study in healthy volunteers, the simultaneous administration of SUG with ROC or VEC evidenced a faster decrease in plasmatic concentrations of $\mathrm{NMB}$ agents than those of SUG facilitating free SUG side effects [14]. This could imply that, in the clinical setting, if SUG is administered at high doses, the unbound SUG molecules will remain free, increasing the possibility of inducing toxic effects [5]. The affinity of SUG for ROC is 2.5 times higher than VEC [18]. In this sense, the rapid onset of action and rapid reversal of effects by SUG makes ROC preferable to VEC $[19,20]$.

Both ROC and VEC have hepatic and renal metabolism [21] and the rate of elimination of NMB depends on their half-life, being shorter for VEC [22]. SUG is eliminated unaltered via urine $[23,24]$ and the inactive complex ROC-SUG or VEC-SUG is rapidly cleared through the kidney, according to the pharmacokinetic properties of SUG $[25,26,5]$. Therefore, when renal or hepatic function is impaired, ROC, VEC and SUG half-life and plasma concentration can be increased [22].

Most frequently reported side effects of SUG administration are pain, nausea, coughing, headache or vomiting $[2,10]$, being the hypersensitivity reactions, ranging from erythema to anaphylactic shock, are the most alarming among them [27,28,29]. Furthermore, allergy to NMB has been considered the first cause of perioperative anaphylaxis $[30,31]$. ROC could induce anaphylactic shock after induction of anaesthesia [32], and patients with a positive history of antibiotic hypersensitivity have a higher risk to develop intraoperative anaphylaxis [33]. ROC also has 
the potential to interact with other drugs, including aminoglycosides [34]. The incidence of VEC-induced anaphylactic reactions is lower than those induced by ROC [28]. Although SUG itself could induce hypersensitivity reactions, it was used to stop anaphylaxis induction by ROC, based on SUG design to encapsulate ROC [35]. In previous studies, SUG has proven capable of reversing the increase in mast-cell number and degranulation caused by ROC [36].

ROC, VEC or SUG cannot pass through a healthy and mature blood-brain barrier (BBB) [37,38]. However, there is evidence that patients with an impaired BBB function treated with either of these compounds, present autonomic dysfunction, seizures [39] and neuronal cell death [15]. Furthermore, ROC, but not VEC, has been detected in cerebrospinal fluid in patients with subarachnoid haemorrhage who underwent neurosurgery $[39,40,41]$. This finding could be attributed to the fact that ROC is more hydrosoluble than VEC, so it may easily arrives to central nervous system when BBB is damaged [42].

In summary, these reports highlight that clinical conditions which compromise the BBB integrity, such as Alzheimer's disease, traumatic brain injury, brain ischemia or haemorrhage, meningitis, or immature nervous system, are susceptible of neurotoxicity induced by these drugs. [43,44,45].

Aging is associated with a decline in physiological function, and this includes drug metabolism [46]. In this sense, it has been reported that recovery periods from ROC and VEC were prolonged in elderly patients [47]. Moreover, when SUG was used for the reversion of the neuromuscular blockade induced by ROC, the recovery, although rapid, was slightly slower in the elderly patients $[48,49]$. Also other authors suggest that SUG, especially for ROC, fails to return to baseline values of EMG in elderly people [40]. Furthermore, there is no data about SUG effects in pregnant women, but in animal studies demonstrated the presence of SUG levels in breast milk [50]. SUG is not recommended for patients under 2 years old because of the lack of available data in this population but the risk of neuronal toxicity should be taken into account for future research [43].

In conclusion, the present study demonstrates that ROC or VEC prevents the increase of CytC, AIF, Smac/Diablo expression and CASP-3 activity induced by recommended doses of SUG in neurons in primary culture. Moreover, both ROC and VEC avoid cell death (apoptosis/necrosis) induced by SUG. This study suggests that it would be desirable to titrate SUG to the minimal effective dose in order to prevent excess of free SUG in plasma that could induce neuronal damage, especially in situations of BBB function impairment.

\section{Acknowledgements}

The authors thank to Generalitat Valenciana and University of Valencia for the financial support (AP 073/09).

\section{Competing Interests}

The authors have declared that no competing interest exists.

\section{References}

1. Claudius C, Garvey LH, Viby-Mogensen J. The undesirable effects of neuromuscular blocking drugs. Anaesthesia. 2009 Mar; 64 (Suppl 1): S10-21. doi: 10.1111/j.1365-2044.2008.05866.x.

2. Kovac AL. Sugammadex: the first selective binding reversal agent for neuromuscular block. J Clin Anesth. 2009; 21: 444-453.

3. Wang $\mathrm{H}$, Liang QS, Cheng LR, et al. Magnesium sulfate enhances non-depolarizing muscle relaxant vecuronium action at adult muscle-type nicotinic acetylcholine receptor in vitro. Acta Pharmacol Sin. 2011; 32:1454-1459.

4. Vorce SP, Mallak CT, Jacobs A. Quantitative analysis of the aminosteroidal non-depolarizing neuromuscular blocking agent vecuronium by LC-ESI-MS: A Postmortem Investigation. J Anal Toxicol. 2008; 32: 422-427.

5. Yang LP and Keam SJ. Sugammadex: a review of its use in anaesthetic practice. Drugs. 2009; 69: 919-942.

6. Bowman WC. Neuromuscular block. British Journal of Pharmacology. 2006; 147 (Suppl 1): S277-286.

7. Lien CA. Development and potential clinical impairment of ultra-short-acting neuromuscular blocking agents. Br J Anaesth. 2011; 107 (Suppl 1): S60-71.

8. Martyn JA, Fagerlund MJ, Eriksson LI. Basic principles of neuromuscular transmission. Anaesthesia. 2009; 64 (Suppl 1): S1-9.

9. Jones RK, Caldwell JE, Brull SJ, et al. Reversal of profound rocuronium-induced blockade with sugammadex: A randomized comparison with neostigmine. Anesthesiology. 2008; 109: 816-824.

10. Lemmens HJ, El-Orbany MI, Berry J, et al. Reversal of profound vecuronium-induced neuromuscular block under sevoflurane anesthesia: sugammadex versus neostigmine. BMC Anesthesiol. 2010; 1: 10-15.

11. Adam JM, Bennett DJ, Bom A., et al. Cyclodextrin-dereved host molecules as reversal agents for the neuromuscular blocker rocuronium bromide: synthesis and structure-activity relationships. J Med Chem. 2002; 45: 1806-1816.

12. Naguib M. Sugammadex: another milestone in clinical neuromuscular pharmacology. Anesth. Analg. 2007; 104: 575-581.

13. Suy K, Morias K, Cammu G, et al. Effective reversal of moderate rocuroniumor vecuronium-induced neuromuscular block with sugammadex, a selective relaxant binding agent. Anesthesiology. 2007; 106: 283-288.

14. Cammu G, De Kam PJ, Demeyer I, et al. Safety and tolerability of single intravenous doses of sugammadex administered simultaneously with rocuronium or vecuronium in healthy volunteers. Br J Anaesth. 2008; 100: 373-379.

15. Palanca JM, Aguirre-Rueda D, Granell MV, et al. Sugammadex, a neuromuscular blockade reversal agent, causes neuronal apoptosis in primary cultures. Int J Med Sci. 2013; 10: 1278-1285.

16. Mosmann T. Rapid colorimetric assay for cellular growth and survival: application to proliferation and cytotoxicity assays. J Immunol Methods. 1983; 65: 55-63.

17. Peterson GL. A simplification of the protein assay method of Lowry et al. which is more generally applicable. Anal Biochem. 1977; 83: 346-356.

18. Akha AS, Rosa J, Jahr JS, et al. Sugammadex: cyclodextrins, development of selective binding agents, pharmacology, clinical development, and future directions. Anesthesiol Clin. 2010; 28: 691-708.

19. Bom A, Hope F, Rutherford S, et al. Preclinical pharmacology of sugammadex. J Crit Care. 2009; 24: 29-35.

20. Haerter F, Eikermann M. Reversing neuromuscular blockade: inhibitors of the acetylcholinesterase versus the encapsulating agents SUG and calibration. Expert Opin Pharmacother. 2016; 17: 819-833.

21. Proost JH, Eriksson LI, Mirakhur RK, et al. Urinary, biliary and faecal excretion of rocuronium in humans. Br J Anaesth. 2000; 85: 717-723.

22. Cammu G, Van Vlem B, van den Heuvel $M$, et al. Dialysability of sugammadex and its complex with rocuronium in intensive care patients with severe renal impairment. Br J Anaesth. 2012; 109: 382-390.

23. Nag K, Singh DR, Shetti AN, et al. Sugammadex: A revolutionary drug in neuromuscular pharmacology. Anesth Essays Res. 2013; 7: 302-306.

24. de Souza CM, Tardelli MA, Tedesco $\mathrm{H}$, et al. Efficacy and safety of sugammadex in the reversal of deep neuromuscular blockade induced by 
rocuronium in patients with end-stage renal disease: A comparative prospective clinical trial. Eur J Anaesthesiol. 2015; 32: 681-686.

25. Peeters P, Passier P, Smeets J, et al. Sugammadex is cleared rapidly and primarily unchanged via renal excretion. Biopharm Drug Dispos. 2011; 32: 159-167.

26. Ren W, Jahr JS. Reversal of neuromuscular block with a selective relaxant binding agent (SRBA). Am J Ther. 2009; 16: 295-299.

27. Jeyadoss J, Kuruppu P, Nanjappa N, et al. Sugammadex hypersensitivity-a case of anaphylaxis. Anaesth Intensive Care. 2014; 42: 89-92.

28. Sadleir $\mathrm{PH}$, Russell $\mathrm{T}$, Clarke $\mathrm{RC}$, et al. Intraoperative anaphylaxis to sugammadex and a protocol for intradermal skin testing. Anaesth Intensive Care. 2014; 42: 93-96.

29. Soria A, Motamed C, Gaouar H, et al. Severe reaction following sugammadex injection: hypersensitivity? J Investig Allergol Clin Immunol 2012; 22: 382.

30. Chiriac AM, Demoly P. Allergy to neuromuscular blocking agents. Presse Med. 2016; 45: 768-773.

31. Takazawa $\mathrm{T}$, Mitsuhata $\mathrm{H}$, Mertes PM. Sugammadex and rocuronium-induced anaphylaxis. J Anesth. 2016; 30: 290-297.

32. Schulberg EM, Webb AR, Kolawole H. Early skin and challenge testing after rocuronium anaphylaxis. Anaesth Intensive Care. 2016; 44: 425-427.

33. Hagau N, Gherman N, Cocis M, et al. Antibiotic-induced immediate type hypersensitivity is a risk factor for positive allergy skin tests for neuromuscular blocking agents. Allergol Int. 2016; 65: 52-55.

34. Hasfurther DL, Bailey PL. Failure of neuromuscular blockade reversal after rocuronium in a patient who received oral neomycin. Can J Anaesth. 1996; 43: 617-620.

35. Barbosa FT, da Cunha RM. Case of anaphylaxis induced by rocuronium treated with sugammadex. Rev Bras Anestesiol. 2012; 62: 538-542.

36. Tomak Y, Yilmaz A, Bostan $\mathrm{H}$, et al. Effects of sugammadex and rocuronium mast cell number and degranulation in rat liver. Anaesthesia. 2012; 67: 1101-1104.

37. Reynolds LM, Lau M, Brown R, et al. Bioavailability of intramuscular rocuronium in infants and children. Anesthesiology. 1997; 87: 1096-1105.

38. Huang S, Kim JK, Atochin $\mathrm{DN}$, et al. Cerebral blood volume affects blood-brain barrier integrity in an acute transient stroke model. J Cereb Blood Flow Metab. 2013; 33: 898-905.

39. Cardone C, Szenohradszky J, Yost S, et al. Activation of brain acetylcholine receptors by neuromuscular blocking drugs. A possible mechanism of neurotoxicity. Anesthesiology. 1994; 80: 1155-1161

40. Fuchs-Buder T, Strowitzki M, Rentsch K, et al. Concentration of rocuronium in cerebrospinal fluid of patients undergoing cerebral aneurysm clipping. Br J Anaesth. 2004; 92: 419-421.

41. Tassonyi E, Fathi M, Hughes GJ, et al. Cerebrospinal fluid concentrations of atracurium, laudanosine and VEC following clinical subarachnoid haemorrhage. Acta Anaesthesiol Scand. 2002; 46: 1236-1241.

42. Roy JJ, Varin F. Physicochemical properties of neuromuscular blocking agents and their impact on the pharmacokinetic-pharmacodynamic relationship. Br J Anaesth. 2004; 93: 241-248.

43. Langley RJ, McFadzean J, McCormack J. The presumed central nervous system effects of rocuronium in a neonate and its reversal with sugammadex. Paediatr Anaesth. 2016; 26: 109-111.

44. Schmidt S, Saunders AM, De La Paz MA, et al. Association of the apolipoprotein $\mathrm{E}$ gene with age-related macular degeneration: possible effect modification by family history, age, and gender. Mol Vis. 2000; 31: 287-293.

45. Rahe-Meyer N, Fennema $H$, Schulman $S$, et al. Effect of reversal of neuromuscular blockade with sugammadex versus usual care on bleeding risk in a randomized study of surgical patients. Anesthesiology. 2014; 121: 969-977.

46. Priebe HJ. The aged cardiovascular risk patient. Br J Anaesth. 2000; 85: 763-778.

47. McDonagh DL, Benedict PE, Kovac AL, et al. Efficacy, safety, and pharmacokinetics of sugammadex for the reversal of rocuronium-induced neuromuscular blockade in elderly patients. Anesthesiology. 2011; 114: 318-329.

48. Leysen J, Bridts CH, De Clerck LS, et al. Rocuronium-induced anaphylaxis is probably not mitigated by sugammadex:evidence from an in vitro experiment. Anaesthesia. 2011; 66: 526-527.

49. Shin S, Han DW, Lee HS, et al. Elderly patients require higher doses of Sugammadex for rapid recovery from deep neuromuscular block. Basic Clin Pharmacol Toxicol. 2016 Jun; 118: 462-467.

50. Merck \& Co Inc. BRIDION: EPAR-product Information Annex I: Summary of product characteristics European Medicines Agency; London, UK: http://www.ema.europa.eu/docs/en_GB/document_library/EPAR_Produc t_Information/human/000885/WC500052310.pdf 